\title{
Modern status of the Tien-Shan cosmic ray station
}

\author{
V.A. Ryabov ${ }^{1, a}$, A.M. Almenova ${ }^{2}$, V.P. Antonova ${ }^{3}$, R.U. Beisembayev ${ }^{1,5}$, S.P. Bezshapov ${ }^{1}$, A.S. Borisov ${ }^{1}$, A.P. \\ Chubenko $^{1}$, O.D. Dalkarov ${ }^{1}$, A.V. Gurevich ${ }^{1}$, A.N. Karashtin ${ }^{4}$, O.N. Kryakynova ${ }^{3}$, G.G. Mitko ${ }^{1}$, R.A. Mukhamedshin ${ }^{10}$, \\ A.M. Mukhashev ${ }^{9}$, R.A. Nam ${ }^{1,5}$, N.F. Nikolaevsky ${ }^{3}$, V.P. Pavlyuchenko ${ }^{1}$, V.V. Piscal ${ }^{1,5}$, M.O. Ptitsyn ${ }^{1}$, V.S. Puchkov ${ }^{1}$, \\ N.O. Saduev ${ }^{8}$, T.Kh. Sadykov ${ }^{2}$, N.M. Salikhov ${ }^{3}$, S.B. Shaulov ${ }^{1}$, A.L. Shepetov ${ }^{1}$, Yu.V. Shlyugaev ${ }^{6}$, A.V. Stepanov ${ }^{1,5}$, \\ W.M. Thu ${ }^{7}$, L.I. Vildanova ${ }^{1,5}$, M.I. Vildanova ${ }^{1,5}$, N.N. Zastrozhnova ${ }^{2}$, V.V. Zukhov ${ }^{1,5}$, and K.P. Zybin ${ }^{1}$ \\ 1 P.N. Lebedev Physical Institute of the Russian Academy of Sciences (FIAN), 119991, Leninskii pr., 53, Moscow, Russia \\ 2 Institute for Physics and Technology, Almaty, 050032, Ibragimova, 11, Kazakhstan \\ ${ }^{3}$ Institute of Ionosphere, 050020, Kamenskoye plato, Almaty, Kazakhstan \\ ${ }^{4}$ Research Radiophysics Institute, 603950, Bolshaya Pechyorskaya, str., 25/12a, Nizhny Novgorod, Russia \\ 5 Tien Shan Mountain Cosmic Ray Station, 050020, Mitina-str., 3, Almaty, Kazakhstan \\ ${ }^{6}$ Institute of Applied Physics of RAS, 603950, Ulyanova str., 46, Nizhny Novgorod, Russia \\ 7 Moscow Institute of Physics and Technology (State University), 117303, Moscow, Russia \\ ${ }^{8}$ Institute for Experimental and Theoretical Physics of al-Farabi Kazakh National University, al-Farabi pr., 71, Almaty, Kazakhstan \\ 9 Abay Kazakh National Pedagogic University, Dostyk pr., 13, Almaty, Kazakhstan \\ ${ }^{10}$ Institute for Nuclear Research, 60-letiya Oktyabrya pr. 7a, 117312 Moscow, Russian Federation
}

\begin{abstract}
We present a description of the new complex installation for the study of extensive air showers which was created at the Tien Shan mountain cosmic ray station, as well as the results of the first measurements made there in 2015-2016. We also present new results on high-energy radiation observed during a thunderstorm.
\end{abstract}

\section{Introduction}

The Tien Shan cosmic ray station is situated in the mountains of Northern Tien Shan (Kazakhstan), $43^{\circ}$ North, $75^{\circ}$ East at an altitude of $3340 \mathrm{~m}$ above sea level. The average atmosphere depth at the height of the station location is $690 \mathrm{~g} / \mathrm{cm}^{2}$. At the station a multifunction complex for investigating the various components of extensive air showers has been created $[1,2]$. The new complex aims to investigate the following fundamental problems in astroparticle and atmospheric physics:

- High-precision measurements of the primary cosmic ray spectrum, mass composition, and distribution of the arrival directions in the range of $E_{0} \sim 10^{14}-10^{17} \mathrm{eV}$ using a wide-spread scintillation shower system, a hybird ionization-neutron calorimeter, muon detectors, Cherenkov radiation detectors;

- Investigation of the parameters of high-energy nuclear interactions, especially shows in the region of EAS cores using a dense carpet of scintillation detectors with an enhanced up to $(1-2) \cdot 10^{6}$ dynamic range of amplitude measurements, in combination with an ionization calorimeter, saturation-free neutron detectors, X-ray films, underground neutron and muon installations;

- A search for strong interacting dark matter particles whose existance in the Universe is supposed to be in the form of exotic nuclei (e.g. strangelets) having a

a e-mail: ryabov@x4u.lebedev.ru specific combination of low charge $Z \leq 10$ and large mass $A \geq 350$;

- Monitoring the intensity of $10^{8}-10^{11} \mathrm{eV}$ cosmic rays of solar origin with application of the low- and highenergy neutron and gamma-radiation detectors; the control for "cosmic weather";

- Investigation of the CR influence on different environmental phenomena; lightning initiation in the atmosphere, intensity of the near-Earth radioactive background, anticipated seismic effects from the passage of powerful EAS, etc.

The Tien-Shan station is a unique place to examine the interconnection between cosmic rays and lightning discharges [3,4]. During a summer season (from the end of May until the beginning of September) thunderstorm clouds pass directly at the height of station. The uniqueness of the experimental conditions and the presence of modern specialized equipment at Tien-Shan provide new data on the processes under investigation. Simultaneous observations of gamma-ray, optical and radio emission, high-energy electrons, positrons, muons and neutrons at high altitudes became a new tool for studying the processes in the thunderstorm atmosphere [5-8].

\section{Instrumentation}

\subsection{EAS Installation}

The new experimental complex includes two systems for precision studies of the shower core, timing system

(c) The Authors, published by EDP Sciences. This is an Open Access article distributed under the terms of the Creative Commons Attribution License 4.0 (http://creativecommons.org/licenses/by/4.0/). 


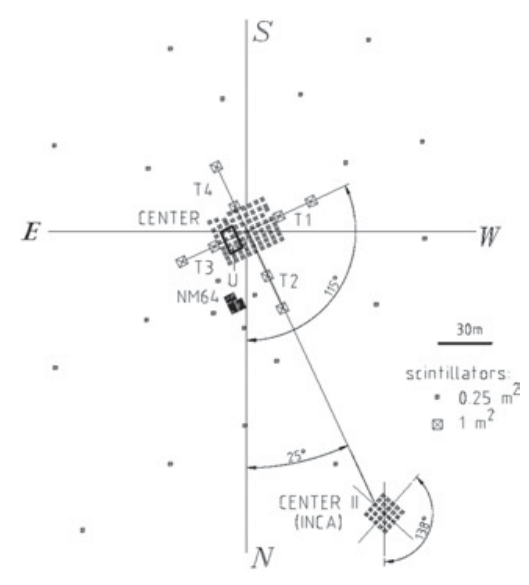

Figure 1. General plan of the Tien Shan EAS detector system.

for determination of the EAS arrival directions, the underground system of muon and hadron detectors for registration of the penetrating components of the shower, ionization-neutron calorimeter and a neutron supermonitor. The layout of the new EAS detector complex on the territory of station is shown in Fig. 1.

The shower system is built around two points, Center and Center II, where the shower core detectors are placed: the dense scintillation "carpet" for registration of the EAS $e / \gamma$ component, a set of timing scintillators T1T4 for determination of the EAS arrival directions according to relative time delays of shower front, the complex of muon and hadron detectors in the underground room, $\mathrm{U}$, situated beneath the Center point; another scintillation carpet, and the big ionization calorimeter INCA in Center II. The NM64 type neutron supermonitor is also placed in the vicinity of the first detector center which is used as a detector of the EAS hadron component. A number of additional scintillation detectors should be spread nearly concentrically over the station territory around the two center cores for registration of the peripheral EAS particles.

At present, the system for registration of the electromagnetic shower component consists of $\sim 100$ detector points built on the basis of plastic scintillator plates with a sensitive area of $0.25 \mathrm{~m}^{2}$ and $1 \mathrm{~m}^{2}$, spread equidistantly over $\sim 10^{4} \mathrm{~m}^{2}$ space. The dynamic range of scintillation amplitude measurements is currently about $(3-7) \cdot 10^{4}$, and there is a prospect of it being extended up to $\sim 10^{6}$. The direction of shower arrival is defined by signal delays from a number of the scintillators placed cross-wise at the periphery of the detector system.

To investigate the nuclear active shower components a multi-tier $55 \mathrm{~m}^{2}$ ionization-neutron calorimeter with a total absorber thickness of $\sim 1000 \mathrm{~g} / \mathrm{cm}^{2}$, a typical spatial resolution of the order of $10 \mathrm{~cm}$, and dynamic range of ionization measurement channel about $\sim 10^{5}$ was created. In addition, the use of saturation-free neutron detectors is anticipated for registration of the high- and low-energy hadron components in the region of the shower core. A complex of underground detectors is designed for the study of muonic and penetrative nuclear active components of the shower.

The full stack of data acquisition, detector calibration, and shower parameters restoration procedures is now completed.

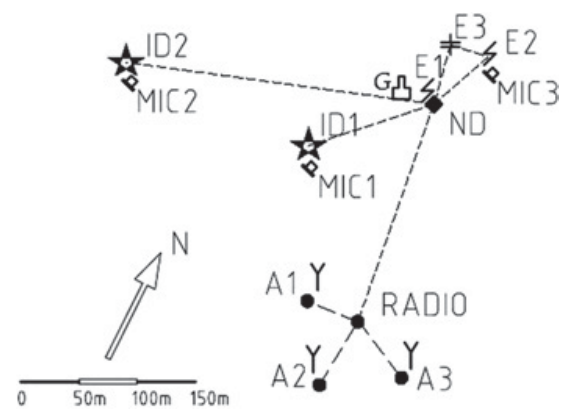

Figure 2. Schematic of the detector complex used in the present experiment. ID1 and ID2 - ionization detectors; Gscintillation detector of the low-energy gamma rays; ND - the neutron detector complex; A1-A3 - radio-antennae; E1-E3 electric field sensors; MIC1-MIC3 - acoustic sensors of the thunder sound.

\subsection{Thunderstorm installation}

The present disposition of the Thunderstorm complex at the Tien-Shan station is shown in Fig. 2.

The "Thunderstorm" complex now comprises the following facilities: an EAS registering system; a system of NaI scintillation detectors for registration of the gamma- and X-ray emission in the atmosphere; the multilayer ionization detectors of energetic charged particles; a neutron super monitor for registration of the highenergy hadronic component of cosmic rays; a set of detectors for low-energy (thermal) neutron background; two independent radio systems; electrostatic detectors of the local electric field and its high frequency component.

\section{Observational results}

\subsection{First EAS measurements}

The integral spectra of the registered EAS over the number of charged particles $N_{e}$ are shown in Fig. 3, where the spectrum obtained in the current experiment is compared with the ones measured at different time by the old shower detector system of the Tien Shan mountain station [9].

The approximation of the Aragats spectrum published in [10] is also shown on this plot. The newly obtained spectrum agrees satisfactorily with other ones, both in its absolute intensity and slope; its slight deviation from the correct pure power form in the range of $N_{e} \geq 2 \cdot 10^{6}$ can be explained by the absence of peripheral shower detectors in our test measurements which resulted in a noticeable side effect with a relative rise of reconstructed $N_{e}$ values when the lateral size of shower is comparable with geometrical size of the whole Center carpet.

As an illustration of the data newly obtained with the neutron calorimeter in the underground room, Fig. 4 shows the spectra of neutron events over the multiplicity of the registered evaporation neutrons $M$ (which is connected with the total energy deposit $E_{h}$ of the initial CR particles inside the detector unit).

The top pair of spectra shown in this plot were calculated for the events registered in either the upper or in the lower unit of the vertical neutron calorimeter under the control of the corresponding internal trigger which has been generated at the moment of the fast transient increase of neutron multiplicity inside the unit; generally, these spectra are similar to old ones published in [11]. Another 


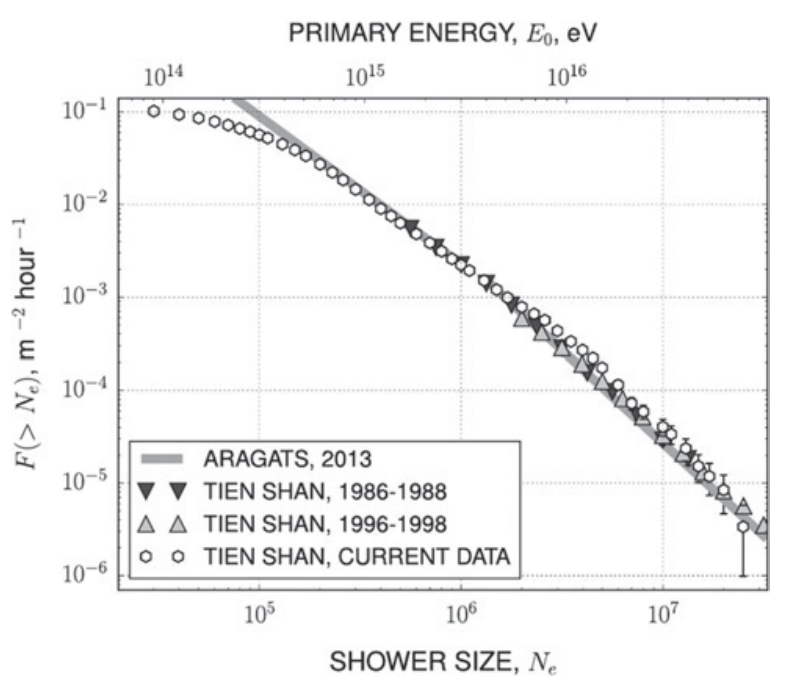

Figure 3. The integral EAS size spectrum obtained at the new shower installation (circles) compared with the spectra measured earlier at Tien Shan [9] (triangles), and Aragats [10] (gray line). For convenience, the upper axis represents the estimations of the corresponding primary energy $E_{0}$ which have been calculated as $E_{0}=3 \cdot 10^{9} N_{e} \mathrm{eV}$.

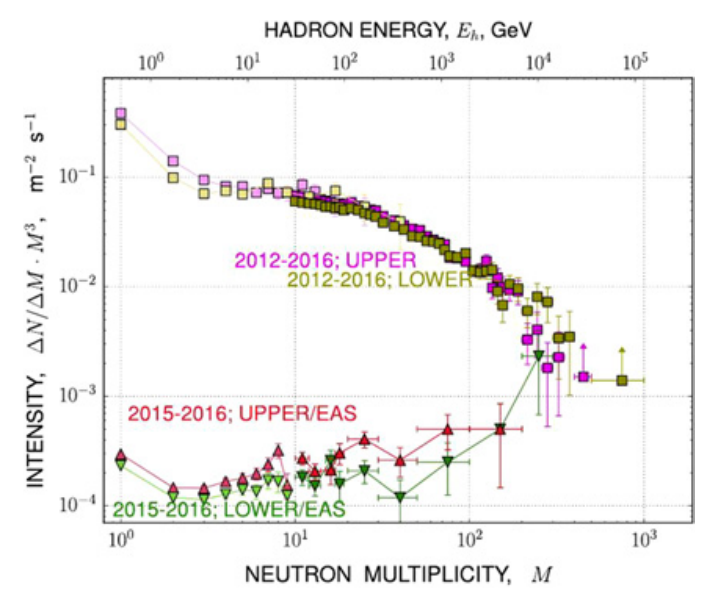

Figure 4. The neutron multiplicity spectra of events registered within the upper and lower units of the vertical underground calorimeter. For reference, the upper axis corresponds to estimations of the hadron energy deposit in the neutron detector unit which have been calculated according to the neutron monitor calibration formula taken from: $E_{h}=0.49 \cdot M^{1.8} \mathrm{GeV}$.

spectra pair with much lower absolute intensity shown at the bottom of Fig. 4 corresponds to the events triggered by EAS passages at the time of the test exploitation of the new shower system in 2015-2016. With an increase of statistics, such data will be useful to clarify the nature of underground neutron events.

\subsection{Observation of high-energy radiation during thunderstorms}

The high-energy radiation observed during a thunderstorm is of two types [8]. One is a long-period emission lasting some tens of minutes or even hours. Another is a short one but very intense. It lasts less than a second, and usually the main part of this emission lasts even a few milliseconds. The emission of the first type is connected with thunderclouds. It is usually supposed to
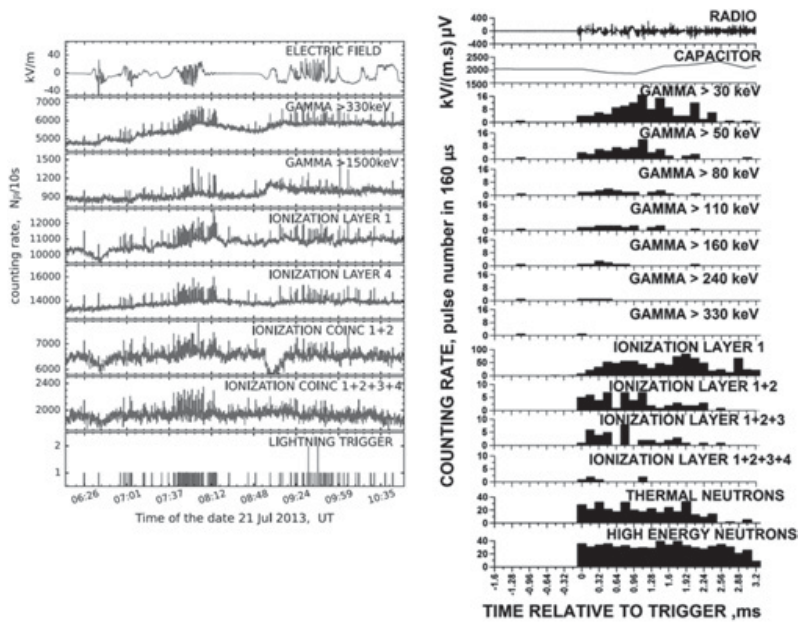

Figure 5. Left (a): monitoring mode with a time resolution of $10 \mathrm{~s}$, Right (b): the triggering mode with a time resolution of $160 \mu \mathrm{s}$. In this figure we present the amplitude of radio emission on the antenna input, the strength of electric field and the counting rates in different types of particle detectors: gamma-rays for different energy thresholds, electrons for coincidences layers in respective energies $([1]-1 \mathrm{MeV},[1+2]-7.6 \mathrm{MeV},[1+2+3]$ $-12 \mathrm{MeV},[1+2+3+4]-16 \mathrm{MeV})$, and also neutrons for thermal $(\sim 0.025 \mathrm{eV})$ and high energy range $(\geq 1 \mathrm{MeV})$.

be determined mainly by the emanation of radioactive gases ( $\mathrm{Rn}$ or $\mathrm{Rn}$ daughter). This long-period emission gives the main input into the high-energy emission of thunderstorms. In Fig. 5a it is shown that lightning could be diagnosed by its high-energy emission in the $10 \mathrm{~s}$ monitoring mode.

For a detailed study of penetrating emissions during thunderstorms, a high-resolution triggering method should be used. The wide complex of all emission type measurements in a $160 \mu$ s triggering mode was fulfilled (Fig. 5b).

The spectrum of lightning gamma emission was obtained. Together with gamma quanta, relativistic highenergy electrons are observed (Fig. 6). The energy spectrum of electrons is registered up to tens of $\mathrm{MeV}$. The gamma and electron high-energy spectra are obtained simultaneously in multiple lightning events. The intensity of both electrons and gamma rays in lightning discharges prevail over the background emission by 1.5 to 2 orders of magnitude. Additionally, the lightning neutron emission was registered. It was shown to consist both of low-energy neutrons and of the intensive flow of energetic neutrons.

\section{Conclusions}

The new complex for investigation of the various components of extensive air showers has been created at the Tien Shan station. Test measurements carried out in 2014-2016 confirmed the efficient operation of the shower detector subsystems of the EAS installation. The results obtained so far demonstrate the reliable measurement of the EAS particle density and shower size spectrum within the energy range of $10^{14}-10^{17} \mathrm{eV}$. High-resolution time series of neutron intensity, and the spectra of energy deposits in the hadron component were registered both on the surface of the Tien Shan station and underground by the neutron monitor like installations used for detecting 


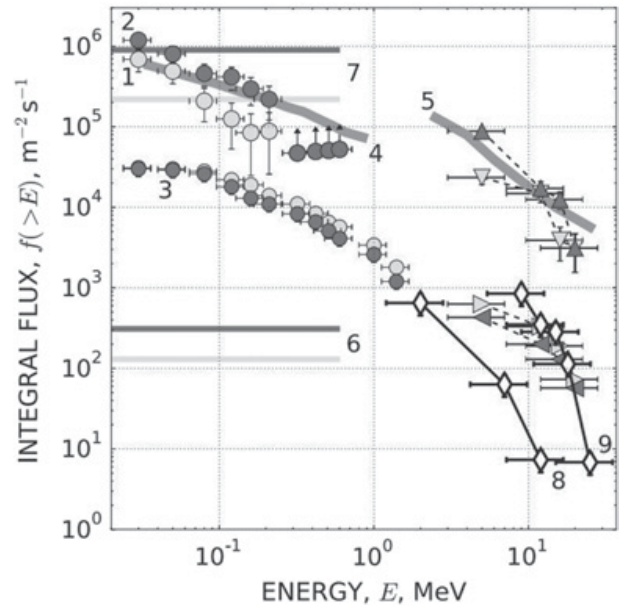

Figure 6. The energy spectra of gamma-radiation (circles) and accelerated electrons (triangles). 1 and 2 - two spectra for the transient radiation bursts around lightning trigger time in the high-resolution series for the two thunderstorm events; - the average spectra calculated over monitoring type measurements at Tien Shan in two thunderstorm days; 4 and 5 - correspondingly, the gamma-ray and electron spectra obtained in Geant4 simulation of an electron avalanche developing in the electric field of thunderclouds; 6 and $7-$ the levels of neutron intensity corresponding to a quite time and just in the moment of transient burst on the two thunderstorm events; 8 and $9-$ electron spectra registered in two thunderstorm events at Aragats [12].

neutron bearing cosmic ray particles. Similar neutron detector technique is planned to be widely applied in the operation of the big hybrid Tien Shan calorimeter INCA. The current development level of different detector subsystems at the Tien Shan station allows us expect new results in various fields of cosmic ray physics in the near future.

The measurements of all types of penetrating emissions were fulfilled during a thunderstorm at the TienShan station. The measurements were made in both the monitoring mode and in the high-resolution trigger mode.
We have demonstrated that the lightning high-energy emission could be directly observed in the 10 s monitoring mode. The results are confirmed by the simultaneous $160 \mu$ s triggering mode measurements. We also showed a significant difference in the magnitudes of penetrating radiation fluxes, depending on the time resolution of the recording apparatus. This integrated approach opens up a new area of research in atmospheric physics at high energies.

\section{References}

[1] A.S. Borisov, A.P. Chubenko, O.D. Dalkarov, et al., Proc. of Science, V. 30-July-2015, Article number 570 (2015)

[2] A.P. Chubenko, A.L. Shepetov, V.P. Antonova, et al., Nuclear Instruments and Methods in Physics Research A 832, 158 (2016)

[3] A.V. Gurevich, A.N. Karashtin, V.A. Ryabov, et al., Phys.-Uspekhi 52(7), 735 (2009)

[4] A.V. Gurevich, G.G. Mitko, V.P. Antonova, et al., Phys.Lett. A 373, 3550 (2009)

[5] A.V. Gurevich, A.N. Karashtin, A.P. Chubenko, et al., Phys.Lett. A325, 389 (2004)

[6] A.V. Gurevich, V.P. Antonova, A.P. Chubenko, et al., Phys. Rev. Lett. 108, 125001 (2012)

[7] A.V. Gurevich, V.P. Antonova, A.P. Chubenko, et al., Phys. Rev. Lett. 111, 165001 (2013)

[8] A.V. Gurevich, A.M. Almenova, V. P. Antonova, et al., Phys. Rev. D 94, 023003 (2016)

[9] V.P. Antonova, A.P. Chubenko, S.V. Kryukov, et al., J. Phys. G 28(2), 251 (2002)

[10] P. Garyaka, R.M. Martirosov, S.O. Sokhoyan, et al., Bull. Natl. Acad. Sci. Armen. Phys. 48, 79 (2013)

[11] A.P. Chubenko, A.L. Shepetov, L.I. Vildanova, et al., Bull. Lebedev Phys. Inst. 34(4), 107 (2007)

[12] A. Chilingarian, B. Mailyan, and L. Vanyan, in 2nd International Cosmic Ray Workshop, Nor-Amberd, Armenia, 151 (2011) 